\title{
ESBRI: A web server for evaluating salt bridges in proteins
}

\author{
Susan Costantini ${ }^{1,2,3}$, Giovanni Colonna ${ }^{2}$ and Angelo M. Facchiano ${ }^{1,2, *}$ \\ ${ }^{1}$ Laboratory of Bioinformatics and Computational Biology, Institute of Food Science, CNR, via Roma $52 \mathrm{~A} / \mathrm{C}, 83100$ Avellino, Italy; \\ ${ }^{2}$ RISCEB, Research Center of Computational and Biotechnological Sciences, Second University of Naples, via Costantinopoli 16, 80138 \\ Naples, Italy; ${ }^{3} \mathrm{CROM}$, Oncology Research Center of Mercogliano, "Fiorentino Lo Vuolo", via Ammiraglio Bianco, 83013 Mercogliano, \\ Italy; Angelo M. Facchiano* - Email: angelo.facchiano@isa.cnr.it; Phone: 390825 299625; Fax: 390825 299813; * Corresponding author
}

received September 16, 2008; revised October 10; accepted October 15, 2008; published November 09, 2008

\begin{abstract}
:
Salt bridges can play important roles in protein structure and function and have stabilizing and destabilizing effects in protein folding. ESBRI is a software available as web tool which analyses the salt bridges in a protein structure, starting from the atomic coordinates. In the case of protein complexes, the salt bridges between protein chains can be evaluated, as well as those among specific charged amino acids and the different protein subunits, in order to obtain useful information regard the protein-protein interaction.
\end{abstract}

Availability: The service is available at the URL: http://bioinformatica.isa.cnr.it/ESBRI/.

Keywords: salt bridges; peptides; proteins; protein-protein interaction; web-software

\section{Background:}

In folded proteins, pairs of oppositely charged residues often interact to form salt bridges. Salt bridges play important roles in many aspects of protein structure and function (i.e. oligomerization, molecular recognition, allosteric regulation, domain motions, flexibility, thermostability and alpha-helix capping). Both experimental and theoretical estimates of the electrostatic free energy contribution of the salt bridges range from being stabilizing, insignificant or destabilizing [1-2]. The formation of salt bridges has been proposed to be a slow step in the protein folding. Concerning the continuum electrostatic calculations, highly stabilizing salt bridges have been reported both within protein monomers and across subunit interfaces. Using site directed mutagenesis, some studies have known that the disruption and the introduction of a salt bridge reduces and increases the stability of the thermophile and mesophile protein, respectively [3]. Some surveys have shown that salt bridges tend to be both more energetically favourable in hydrophilic environments or at the surface of proteins, and destabilizing in the center of a protein [4]. However, there are a large number of "buried" salt bridges being energetically favourable for the overall protein and the single most important factor in determining their contribution to a protein's overall stability is the geometry of the interaction [4].

A salt bridge is constituted by a couple of oppositely charged groups, so in proteins it is recognized if at least one Asp or Glu side-chain carboxyl oxygen atom (i.e. OD in Asp or $\mathrm{OE}$ in Glu) and one side-chain nitrogen atom of Arg, Lys or His (i.e. NH in Arg, NZ in Lys or NE \& ND in His) are within a distance of 4.0 Angstroms [1-2]. ESBRI is a user-friendly tool that can analyse any peptide or protein structure, obtained from experimental data or

ISSN 0973-2063 (online) 0973-2063 (print)

Bioinformation 3(2): 137-138 (2008) modelling studies or molecular dynamics simulations. It evaluates all the salt bridges in the whole protein structure or in a single protein chain, among complexed chains and those between user-specified charged residues and the rest of protein. Moreover, in ESBRI the threshold distance between two oppositely charged residues can be modified in order to verify if two oppositely charged residues are at a distance higher/lower than 4 Angstroms.

\section{Methodology:}

Program input

The user can indicate the protein name, paste the coordinates file (in PDB format) in the box and choose five possible analysing options to evaluate: (1) all salt bridges in the peptide or protein structure; (2) the salt bridges present in a specific chain chosen from the user; (3) the salt bridges between two complexed chains indicated from the user; (4) the salt bridges between a positively charged residue (i.e. Arg, Lys or His) and the rest of the structure; (5) the salt bridges between a negatively charged residue (i.e. Glu or Asp) and the rest of the structure. In the fourth and fifth options the user may indicate the "type residue" choosing between those possible, the chain in which it's located and its "residue number". By default settings, the threshold distance to find a salt bridge is fixed at 4.0 Angstroms. As additional options, the user can also modify the threshold distance between two oppositely charged residues, and evidence in the result table the differently charged amino acids by using a color code (blue for positively charged residues, red for negatively charged residues).

\section{Program output}

In the first option ESBRI shows the table with all the putative salt bridges in the analysed structure. The table 
reports the atom types of two residues involved in salt bridge and their distance, expressed in Angstroms. When the user chooses the second option, ESBRI reports in the table only the possible salt bridges in the chain chosen from the user (Figure 1a). In the results page of the third option ESBRI shows the salt bridges between two protein chains chosen from the user and the related distances between the atoms involved in the bonds (Figure 1b). This option allows the user to obtain quickly useful information regarding protein binding sites, protein interfaces, and structural characteristics of protein interactions. When the user chooses the fourth or fifth options, ESBRI reports in table the putative salt bridges between the positively or negatively charged residue, respectively, chosen from the user and the rest of the chain in which it's located or other chains present in the structure file (Figure 1c). This option allows to identify if a specific charged amino acid has a stabilizing effect in its protein chain or if its presence can stabilize the interaction with other proteins. For these reasons, ESBRI can be useful in protein residue mutations and protein-protein interactions studies. The results appear in few seconds and are presented in a HTML page. The user can also save directly the results in text format.

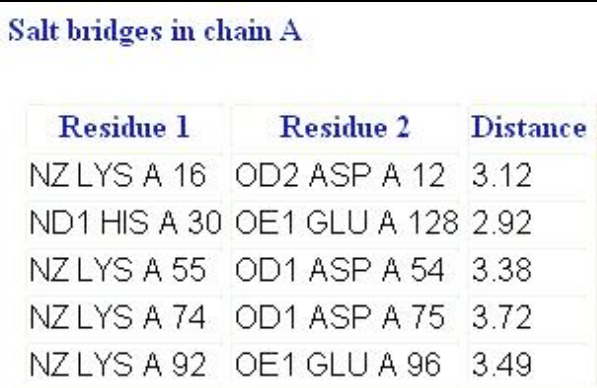

Salt bridges between chain $R$ and chain $L$ :

Residue 1 Residue 2 Distance

NH1 ARG R 76 OD1 ASP L $42 \quad 3.73$

NZLYSL 34 OE1 GLUR 1012.70

Salt bridges for residue GLU A 96

Residue 1 Residue 2 Distance OE1 GLU A 96 NZLYS A 923.49

Figure 1: Example of output results for second (a), third (b) and fifth (c) options.

\section{Caveats and future development:}

ESBRI is a CGI script written in Perl language. We are developing an interface to integrate ESBRI and other tools developed in our lab within a unique web service.

\section{Acknowledgment:}

This work was partially supported by the CNRBioinformatics Project, "RNBIO - Rete Nazionale di Bioinformatica Oncologica” Conv. no. ACC2/R7.11, and Programma Italia-USA "Farmacogenomica Oncologica" Conv. no. 527/A/3A/5.

\section{References:}

[01] S. Kumar and R. Nussinov, J. Mol. Biol., 293: 1241 (1999) [PMID: 10547298]

[02] S. Kumar and R. Nussinov, Biophys J., 83: 1595 (2002) [PMID: 12202384]

[03] S. Kumar et al., Proteins, 38: 368 (2000) [PMID: 10707024]

[04] J. N. Sarakatsannis and Y. Duan, Proteins, 60: 732 (2005) [PMID: 16021620]

Edited by I. Roterman

Citation: S. Costantini et al., Bioinformation 3(2): 137-138 (2008)

License statement: This is an open-access article, which permits unrestricted use, distribution, and reproduction in any medium, for non-commercial purposes, provided the original author and source are credited. 